Original Paper http://ajol.info/index.php/ijbcs http://indexmedicus.afro.who.int

\title{
Contribution de la production anacardière aux moyens de subsistance des ménages Balantes de Mansoa (région d'Oio, Guinée-Bissau)
}

\author{
Mamadou KAMBAYE $^{1^{*}}$, Ngor NDOUR ${ }^{1}$ et Maurice DASYLVA ${ }^{2}$ \\ ${ }^{1}$ Département d'Agroforesterie, Laboratoire d'Agroforesterie et d'Ecologie (LAFE), Université Assane SECK \\ de Ziguinchor, BP 523 Ziguinchor, Sénégal. \\ ${ }^{2}$ Ecole Supérieure des Sciences Agricoles et de l'Alimentation (ES2A), Université Amadou Mahtar MBOW, BP \\ 45927 Dakar Nafa VDN, Sénégal. \\ *Auteur correspondant ; E-mail : kambayemamadou89@gmail.com ; Tél. : +221778101981
}

Received: 08-12-2020

Accepted: 15-04-2021

Published: 30-04-2021

\section{RESUME}

L'agriculture Bissau-guinéenne est actuellement dominée par la production anacardière. Cette dernière, bien que peu étudiée, participe à l'amélioration du cadre de vie et de l'économie locale. Dans le but de contribuer à une meilleure connaissance des impacts du système de production anacardière sur les ménages, l'étude a porté sur 77 ménages répartis dans trois villages Balantes. Sur la base d'enquêtes agro-socioéconomiques, l'étude montre que l'héritage $(73,2 \%)$ est le mode d'accès aux terres gérées que par des hommes. Le semis direct (77\%) et les plants produits en pépinière (33\%) constituent les modes de plantation des anacardiers. Les sous-produits de l'anacardier jouent un rôle alimentaire et commercial pour les ménages. Les noix brutes sont destinées à la vente $(100 \%)$ et celles grillées sont utilisées dans l'alimentation familiale $(100 \%)$. Le jus de cajou est exclusivement réservé à la consommation familiale alors que le vin est réservé pour une grande part à la vente $(52,65 \%)$. Le bois mort est utilisé comme combustible $(52,3 \%)$ et/ou pour la carbonisation destinée à la vente $(47,7 \%)$. D'au final, les revenus tirés du cajou destinés principalement à l'achat du riz $(15,75 \%)$, du matériel agricole $(14,7 \%)$ et au paiement de main d'œuvre rizicole (14,6\%) sont en moyenne estimés à 286770 FCFA/ménage/an. Les revenus engendrés par la culture de l'anacarde permettent aux producteurs de subvenir à leurs besoins, d'améliorer leurs conditions et cadre de vie.

(c) 2021 International Formulae Group. All rights reserved.

Mots clés : Agriculture, plantation, Cajou, impacts.

\section{Contribution of cashew nut production to the livelihoods of Balante households in Mansoa (Oío region, Guinea-Bissau)}

\begin{abstract}
Agriculture in Guinea-Bissau is currently dominated by cashew nut production. The latter, although little studied, contributes to the improvement of the living environment and the local economy. In order to contribute to a better understanding of the impacts of this production system, the study covered 77 households in three Balante's villages. On the basis of agro-socio-economic surveys, the study shows that inheritance $(73.2 \%)$ is the only mode of access to land managed by men. Direct seeding (77\%) and nursery grown plants (33\%) are the most common methods of planting cashew trees. Cashew by-products play a food and commercial role for
\end{abstract}


households. The raw nuts are intended for sale (100\%) and roasted nuts are used in family food (100\%). Cashew juice is exclusively reserved at the family consumption while wine is reserved for a large part for sale $(52.65 \%)$. Dead wood is used as fuel $(52.3 \%)$ and/or for carbonization for sale $(47.7 \%)$. In the end, the income from cashew mainly used to buy rice (15.75\%), agricultural equipment (14.7\%) and to pay for rice labor (14.6\%) is on overage, they are estimated at $286770 \mathrm{~F} \mathrm{CFA/Household/year.} \mathrm{The} \mathrm{income} \mathrm{generated} \mathrm{by} \mathrm{cashew} \mathrm{nut} \mathrm{cultivation} \mathrm{allows}$ producers to meet their needs, improve their living conditions and environment.

(C) 2021 International Formulae Group. All rights reserved.

Keywords : Agriculture, plantation, Cashew, impacts.

\section{INTRODUCTION}

L'agriculture contribue pour environ $52 \%$ du PIB en Guinée-Bissau dont plus de $50 \%$ des produits d'exportation et constitue l'activité économique majeure et la principale richesse du pays (MSI, 2010). La production anacardière est l'une des cultures dominantes en Guinée-Bissau (Bappah et al., 2018). Le cajou y génère $18 \%$ du PIB, $90 \%$ des recettes d'exportation et $33 \%$ des revenus des ménages (Cabral, 2015). L'anacardier se trouve aujourd'hui presque partout dans le pays ; particulièrement au nord (Cacheu, Oio et Bafata), disposant, au plan climatique, des conditions les meilleures (NEPAD, 2006). L'avènement de production anacardière en Guinée-Bissau se traduit par une occupation des terres arables et certains secteurs forestiers, en raison de l'extension des plantations d'anacarde (Pennober, 1999). Pour ce qui est de l'accès à la terre chez les Balantes, c'est au moment où les fils se marient qu'ils reçoivent, du chef de ménage, leurs parcelles de terre (Ribeiro, 2002). En cas de décès du père de famille, c'est le plus âgé des hommes qui assure la gestion des intérêts collectifs du ménage (Ribeiro, 2002). Lors de la mise en place de nouvelles plantations, le semis direct, qui consiste à mettre une ou deux noix par poquet, constitue la principale méthode de plantation (Balogoun et al., 2014; Koffi et Oura, 2019). Selon Ricau (2013), la production des noix d'anacarde en Guinée-Bissau s'étend de mars à juin avec des pics aux mois d'avril et de mai. La culture d'anacardier a été mise en place dans le but de résoudre les problèmes majeurs des paysans, à savoir, l'amélioration de la condition économique et du cadre de vie (Koffi et Oura, 2019). Le jus extrait de la pomme de l'anacardier permet la fabrication du vin qui procure des revenus substantiels aux producteurs (Ndiaye et al., 2017). L'anacardier est une source de bois d'énergie, de charbon de bois qui procure des revenus non négligeables aux ménages, mais aussi de bois de menuiserie (Daffé, 2013). L'écorce macérée dans l'eau est utilisée pour traiter la dysenterie et le rhume (Daffé, 2013). Selon Sinan et Abou (2016), l'anacarde permet aux paysans de célébrer leur mariage, d'acheter du bétail (bœufs) et des moyens de déplacement (motos et vélos) mais aussi des panneaux solaires pour l'électrification rurale. Par ailleurs, les agriculteurs investissent aussi dans la production du riz par la prise en charge du paiement et de l'alimentation des groupes de travail avec les revenus tirés du cajou (Temudo et Abrantes, 2014). Quant aux charges familiales, la culture d'anacardier permet aux paysans de subvenir à leurs besoins sociaux comme l'éducation et la santé de la famille ainsi que la construction d'habitats améliorés (Yabi et al., 2013; Sinan et Abou, 2016). En Guinée-Bissau, la commercialisation de la noix de l'anacarde permet aux paysans de se procurer du riz, principale denrée de consommation des populations (Camara et al., 2007).

Cependant, bien qu'il soit un secteur très rentable dans ce pays, le système de production anacardière reste confronté à des difficultés telles que : le manque de moyens de production modernes, l'inexistence d'une assistance technique suffisante de la part du gouvernement, le non-respect des techniques sylvicoles appropriées recommandées (telles que l'écartement entre les plants, les pratiques d'élagage, la préparation des sols), l'utilisation 
des techniques de récolte et de post-récolte inappropriées (Lo et al., 2016).

C'est dans ce contexte que s'inscrit cette étude qui vise la caractérisation socioéconomique des plantations d'anacarde dans trois villages Balantes de Mansoa pour contribuer à une meilleure connaissance de leurs impacts socio-économiques sur les ménages des producteurs.

\section{MATERIEL ET METHODES}

L'étude porte sur la contribution des produits de l'anacardier aux moyens de subsistance des ménages balantes des villages de Cuboi, N'Tchugal et Cambia-Psora localisés dans le secteur correspond au département dans la subdivision administrative bissauguinéenne.de Mansoa (région administrative de Oio).

\section{Présentation de la zone d'étude}

La zone d'étude est limitée au nord par les secteurs de Mansaba et de Bissora, au sud par le Rio Géba, à l'ouest par le secteur de Nhacra et à l'est par le secteur de Bambadinca dans la région de Bafata (Figure 1). Elle est située à $60 \mathrm{~km}$ de Bissau avec environ 47000 habitants et l'ethnie prédominante est celle des Balantes (Benzinho \& Rosa, 2018). Dans cette zone, le relief est relativement peu accentué, les sols sont pour la majorité, ferralitiques et ferrugineux tropicaux (Ira et al., 2019). L'agriculture, l'élevage, la pêche de subsistance et l'exploitation des produits forestiers ligneux et non ligneux y sont les principales activités socioéconomiques (MADR, 2010).

\section{Méthodes de collecte des données}

Les méthodes de recherche déployées pour atteindre les objectifs de cette étude sont notamment liées aux enquêtes agrosocioéconomiques auprès des ménages.

\section{Plan de sondage}

Il a consisté essentiellement au choix des sites et ménages, à la collecte et au traitement des données.

\section{Choix des sites et ménages}

Le choix des trois villages échantillons se justifie d'une part par le fait que c'est la zone d'intervention du projet (DEDURAM) et de l'autre par la représentativité des plantations d'anacarde dans ces villages de la région ciblée. La taille de l'échantillon a été déterminée sur la base du nombre total de ménages des trois villages (taille de la population) et à l'aide de la formule de Fischer. Selon les recensements effectués par Universsel (2018), le nombre total de ménages dans ces trois villages est de 320. Ainsi, la taille de l'échantillon a été déterminée comme il suit $: \mathrm{nf}=n(1+n / N)$ avec $n=1 / d^{2} ;$ avec: $\mathrm{nf}=$ taille de l'échantillon ; $d=$ degré d'erreur $=10 \% ; \mathrm{N}$ $=$ nombre total des ménages. Application: $\mathrm{n}$ $=1 / 10-2=100$ donc $\mathrm{nf}=100 /(1+100 / 320)=$ $76,19 \approx 77$ ménages à interroger.

Pour la détermination du nombre de ménages à interroger par village, il a procédé par une allocation au prorata de la taille du village en fonction du nombre de ménages (Tableau 1) sur la base de la formule suivante : Pour le village Cuboi : $\mathrm{w}=133 * 77 / 320=$ 32,0031

$\mathrm{w}=32$ avec $\mathrm{w}$ le nombre de ménages à interroger.

Pour le village Cambia-Psora : $\mathrm{x}=109 * 77 / 320$ $=26,2281$

$\mathrm{x}=26$ avec $\mathrm{x}$ le nombre de ménages à interroger.

Pour le village N'Tchugal : $\mathrm{y}=78 * 77 / 320=$ 18,7687

$\mathrm{y}=19$ avec y le nombre de ménages à interroger.

Dans chaque ménage, seul le chef d'exploitation est interrogé qu'il soit homme ou femme.

\section{Les enquêtes agro-socioéconomiques}

Les enquêtes ont été réalisées sur la base d'un questionnaire portant sur des rubriques relatives à l'identification des producteurs, aux systèmes de production anacardière et aux profits générés par les plantations d'anacarde. Dans chaque village, une mission de préenquêtes a été réalisée afin d'établir un 
contact avec les autorités locales, coutumières et administratives. Elle a permis de les informer de l'objet du travail de recherche dans la localité. Les enquêtes individuelles ont été faites avec les producteurs reconnus comme chefs d'exploitations au sein des ménages. Ce choix orienté a conduit à un échantillon de soixante-dix-sept (77) personnes repérées dans les trois villages par la méthode du plus proche voisin. La procédure consiste à repérer un chef d'exploitation avec l'appui d'une personne ressource comme le chef du village ou un membre du projet qui connaît bien les producteurs de la zone d'étude.

\section{Traitement et analyse des données}

Les données collectées sont traitées à l'aide du logiciel Sphinx Plus ${ }^{2}$ (V5). Il a permis de réaliser des analyses uni-variées portant sur les impacts socioéconomiques de la production anacardière sur les ménages des producteurs et de déterminer les tendances centrales des variables. Les statistiques obtenues ont été exportées dans le tableur Excel pour la réalisation et la finalisation des graphiques.

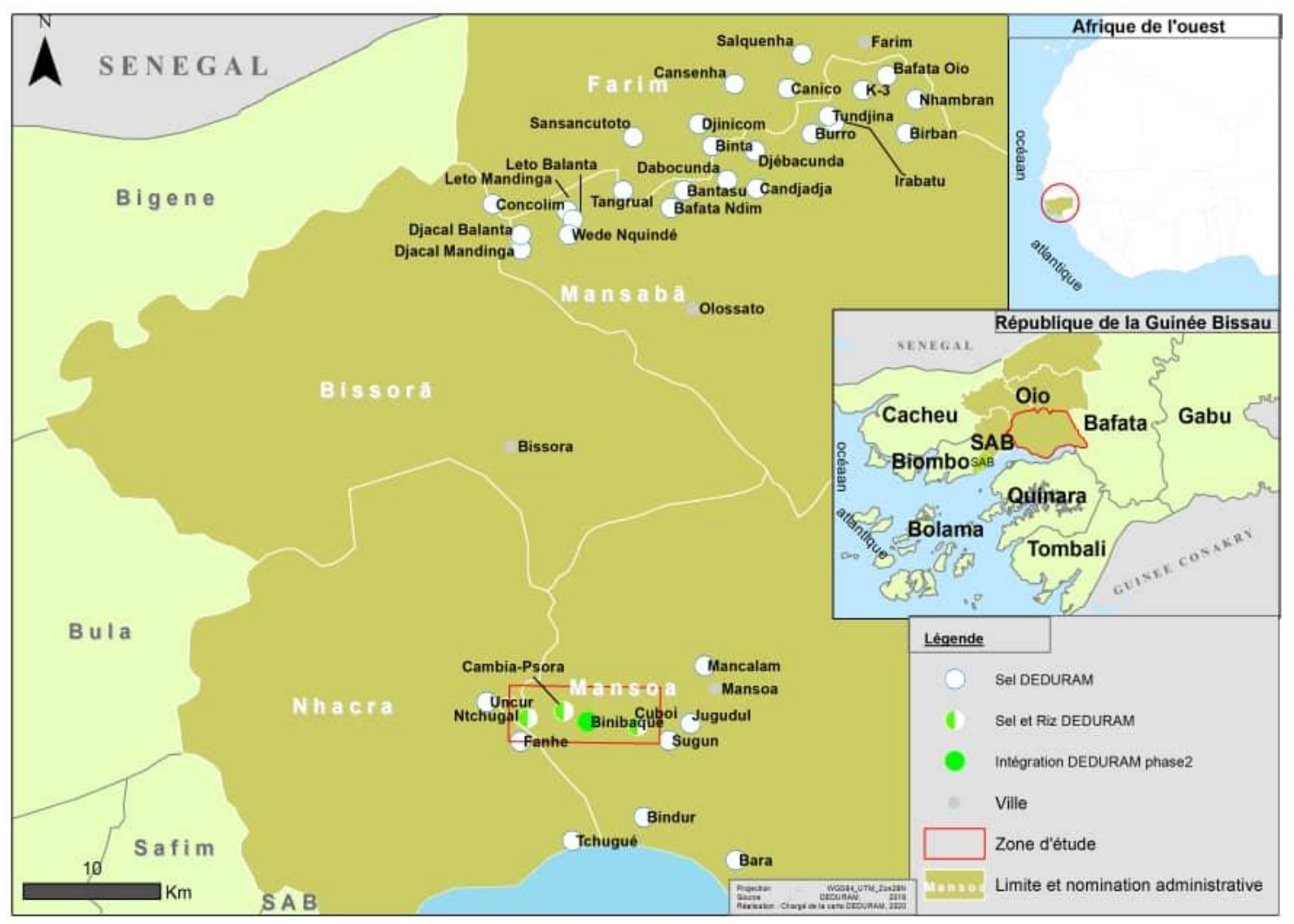

Figure 1 : Carte de localisation des sites de l'étude dans le secteur de Mansoa, région d'Oio.

Tableau 1 : Répartition des ménages enquêtés suivant les villages.

\begin{tabular}{lcc}
\hline Villages & Population (nombre de ménages) & Taille échantillon \\
\hline Cuboi & 133 & 32 \\
Cambia-Psora & 109 & 26 \\
N'Tchugal & 78 & 19 \\
Total & $\mathbf{3 2 0}$ & $\mathbf{7 7}$ \\
\hline
\end{tabular}




\section{RESULTATS}

\section{Caractérisation des producteurs et tenure foncière}

L'analyse des données de l'enquête montre que les enquêtés dans les trois villages sont tous du genre masculin (100\% hommes).

L'analyse de la Figure 2 révèle que $51,3 \%$ des enquêtés ont un âge compris entre 18 - 43 ans avec un âge moyen de 47 ans. Cette classe est suivie par celles de $43-68$ ans $(46,1 \%)$ et de $68-83$ ans avec $2,6 \%$.

L'analyse de la Figure 3 montre que l'héritage est le principal mode d'obtention de la terre $(73,2 \%)$ suivi du droit de hache $(9,8 \%)$; le don et l'achat avec $8,5 \%$ pour les deux.

\section{Mise en place des plantations d'anacarde et calendrier de production}

Selon la majorité des enquêtés $(77,0 \%)$, le semis direct est le principal mode de plantation des champs d'anacarde. Ceux qui déclarent que les plantations sont issues de plants des pépinières représentent $23 \%$ des producteurs (Figure 4).

Le Tableau 2 révèle que la majorité des enquêtés fait le défrichage et le nettoyage entre les mois de Janvier, Février et de Mars avec respectivement $21,1 \%, 36,4 \%$ et $27,1 \%$ des cas. En dehors de cette période, ces travaux se font également aux mois d'Avril, Novembre et de Décembre avec de faibles fréquences de citation respectivement de $9,3 \%, 1,7 \%$ et $4,2 \%$ des cas. La période de la récolte et vente du cajou s'étale du mois de Mars au mois de Juin mais avec une fréquence de citation plus grande pour les mois de Mai et Juin $(30,4 \%)$.

\section{Usages de la main d'œuvre dans les champs d'anacarde}

La Figure 5 révèle que les travaux de défrichage et de nettoyage mobilisent plus de main d'œuvre $(94,8 \%)$. Les producteurs font également appel à la main d'œuvre pour la récolte et vente $(5,2 \%)$.

\section{Usages et destinées des produits tirés de l'anacardier}

Le Tableau 3 permet de voir que les noix brutes sont exclusivement vendues alors que celles grillées sont destinées totalement à la consommation familiale. Concernant le jus obtenu à partir de la pomme, il est seulement utilisé pour la consommation familiale alors que le jus fermenté (vin) est utilisé à 47,35\% pour la consommation de la famille et à $52,65 \%$ pour la vente. Cependant la pomme est également utilisée à $0,6 \%$ pour la fabrication du savon par les femmes. Le bois mort provenant des pieds sénescents ou morts, de l'ébranchage est utilisé comme bois de chauffe $(52,3 \%)$ ou pour la fabrication du charbon destiné à la vente (47,7\%). Dans le domaine de la pharmacopée, le Tableau 3 révèle que les feuilles, les racines et l'écorce sont utilisées avec respectivement $7,9 \% ; 1,3 \%$ et $2,6 \%$ pour les soins médicaux de la famille.

\section{Commercialisation des produits de cajou}

La Figure 6 révèle que les principaux acheteurs de noix sont les "bana-bana" (pisteurs), les Maures et les Indiens mais avec une fréquence de citation plus grande pour les pisteurs $(62,0 \%)$. Pour ce qui est du vin, il est vendu dans la zone de production $(62,5 \%)$, à la population de Mansoa (29,2\%) et à celle de Bissau (8,3\%). Le charbon de bois est principalement vendu à la population de Bissau $(72,7 \%)$ et aux voyageurs $(25,5 \%)$.

La Figure 7 montre que le prix de vente du kg de noix est de 350 FCFA $(66,7 \%)$ avec le prix moyen de 360 FCFA. Par contre les prix de 400 FCFA, 500 FCFA et plus sont aussi appliqués avec respectivement $(22,7 \%)$; $(2,6 \%)$ et $(1,3 \%)$. Cette différence de prix s'explique selon les producteurs par le rapport entre l'offre et la demande et/ou selon la période de vente du produit. Concernant le vin de cajou, le prix de vente est de 100 FCFA le litre $(96,9 \%)$ contre 50 FCFA $(3,1 \%)$ avec un prix moyen de 75 FCFA. Pour le charbon, 2500 FCFA est le prix de vente du sac $(87,2 \%)$ avec un prix moyen de 1900 FCFA. D'autres prix ont été appliqués pour le charbon : 2000 FCFA $(7,7 \%) ; 1500$ et 3000 FCFA $(2,6 \%)$ pour chacun.

\section{Destinées des revenus tirés de la production}

Le revenu moyen tiré de la production anacardière est de 286770 FCFA par ménage et par saison pour l'année 2019. 
Le Tableau 4 montre que les revenus tirés du cajou ont plusieurs destinations. L'achat de riz et du matériel de travail des champs sont plus représentés avec respectivement $15,75 \%$ et $14,7 \%$. Certains producteurs investissent en retour dans les travaux rizicoles $(14,6 \%)$. Par ailleurs, l'achat de fertilisants et de champs de riz ont été cités mais avec de faibles pourcentages $1,65 \%$ et $2,6 \%$ respectivement.

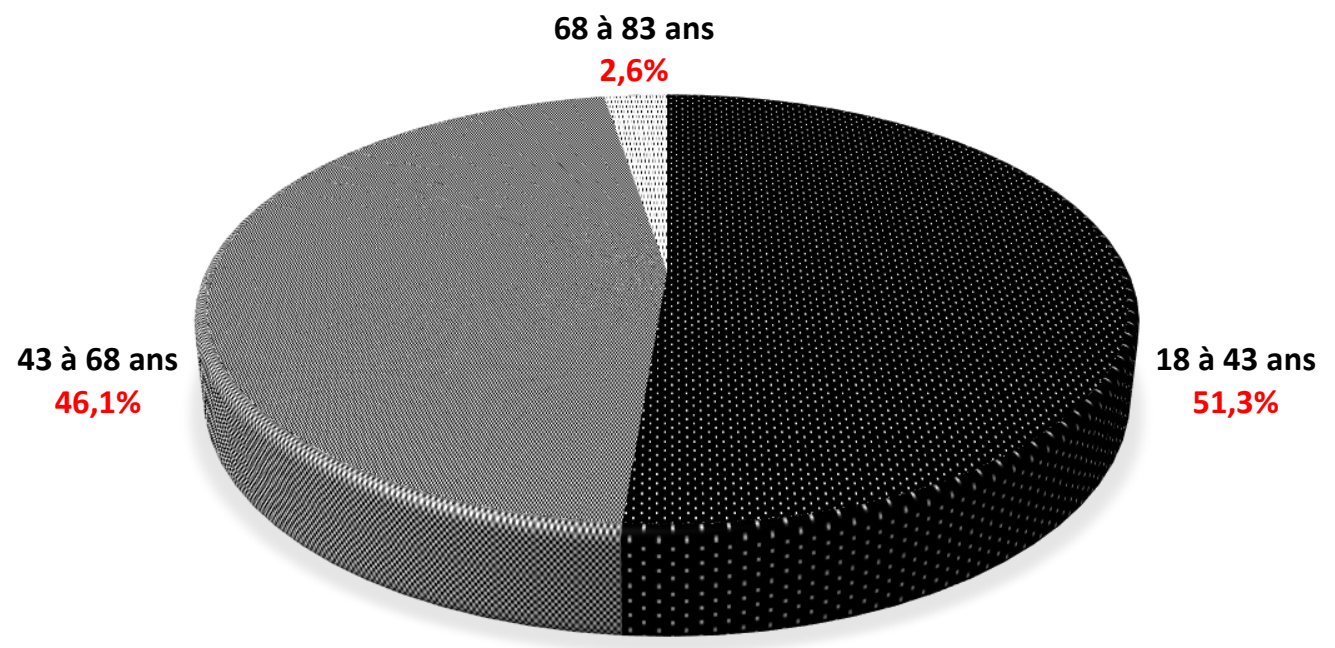

Figure 2 : Répartition des producteurs en fonction des classes d'âge.

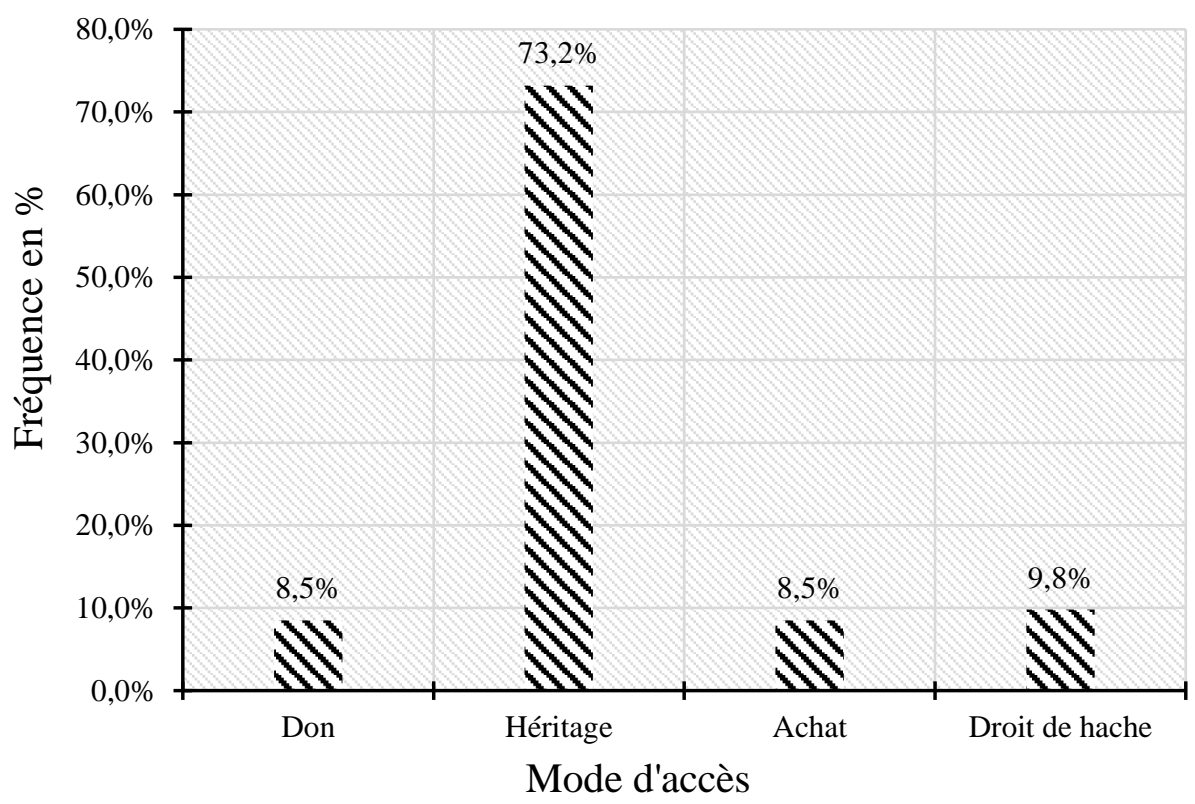

Figure 3 : Fréquences de citation des modes d'accès à la terre. 


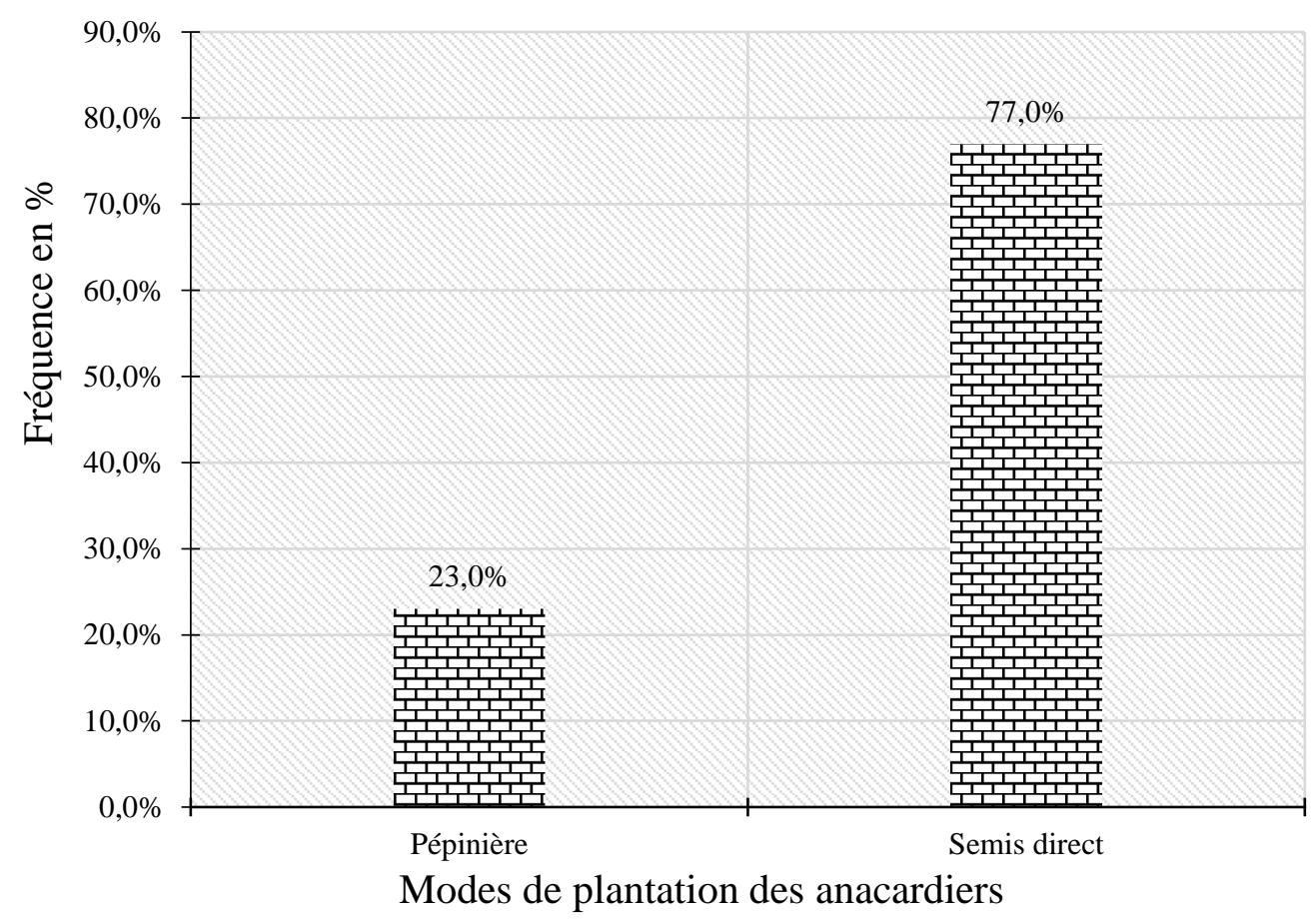

Figure 4 : Fréquences de citation des modes de plantation des anacardiers.

Tableau 2 : Calendrier des activités effectuées dans les plantations d'anacarde.

\begin{tabular}{lcc}
\hline \multirow{2}{*}{ Périodes/mois } & \multicolumn{2}{c}{ Principales tâches effectuées dans les champs d'anacarde } \\
\cline { 2 - 3 } & Défrichage et nettoyage (\%) & Récolte et vente (\%) \\
\hline Janvier & $21,10 \%$ & - \\
Février & $36,40 \%$ & - \\
Mars & $27,10 \%$ & $10,40 \%$ \\
Avril & $9,30 \%$ & $28,80 \%$ \\
Mai & - & $30,40 \%$ \\
Juin & - & $30,40 \%$ \\
Juillet & - & - \\
Août & - & - \\
Septembre & - & - \\
Octobre & - & - \\
Novembre & $1,70 \%$ & - \\
Décembre & $4,20 \%$ & - \\
\hline
\end{tabular}

Le tiré (-) : signifie absence de l'activité précisée durant le mois correspondant. 


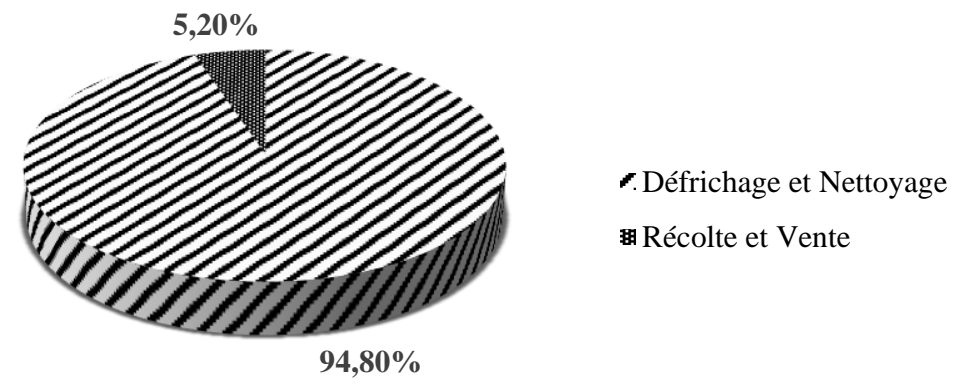

Figure 5 : Répartition de la main d'œuvre en fonction des tâches agricoles.

Tableau 3 : Proportion suivant les catégories d'usage des produits tirés de l'anacardier.

\begin{tabular}{|c|c|c|c|}
\hline $\begin{array}{l}\text { Différents } \\
\text { produits }\end{array}$ & $\begin{array}{c}\text { Nature/état du } \\
\text { produit }\end{array}$ & Destinataire & $\begin{array}{l}\text { Fréquence de } \\
\text { citation }\end{array}$ \\
\hline \multirow[t]{2}{*}{ Noix } & Noix grillées & $\begin{array}{l}\text { Consommation } \\
\text { familiale }\end{array}$ & $100 \%$ \\
\hline & Noix brutes & Vente & $100 \%$ \\
\hline \multirow[t]{4}{*}{ Pomme } & Jus de cajou & $\begin{array}{l}\text { Consommation } \\
\text { familiale }\end{array}$ & $100 \%$ \\
\hline & \multirow[t]{2}{*}{ Vin de cajou } & $\begin{array}{l}\text { Consommation } \\
\text { familiale }\end{array}$ & $47,35 \%$ \\
\hline & & Vente & $52,65 \%$ \\
\hline & Saponification & Utilisation familiale & $0,60 \%$ \\
\hline \multirow[t]{2}{*}{ Bois mort } & Carbonisation & Vente & $47,7 \%$ \\
\hline & Bois d'énergie & Cuisine & $52,3 \%$ \\
\hline Feuilles & Pharmacopée & $\begin{array}{l}\text { Soins médicaux de } \\
\text { la famille }\end{array}$ & $7,90 \%$ \\
\hline Racines & Pharmacopée & $\begin{array}{l}\text { Soins médicaux de } \\
\text { la famille }\end{array}$ & $1,30 \%$ \\
\hline Ecorce & Pharmacopée & $\begin{array}{l}\text { Soins médicaux de } \\
\text { la famille }\end{array}$ & $2,60 \%$ \\
\hline
\end{tabular}




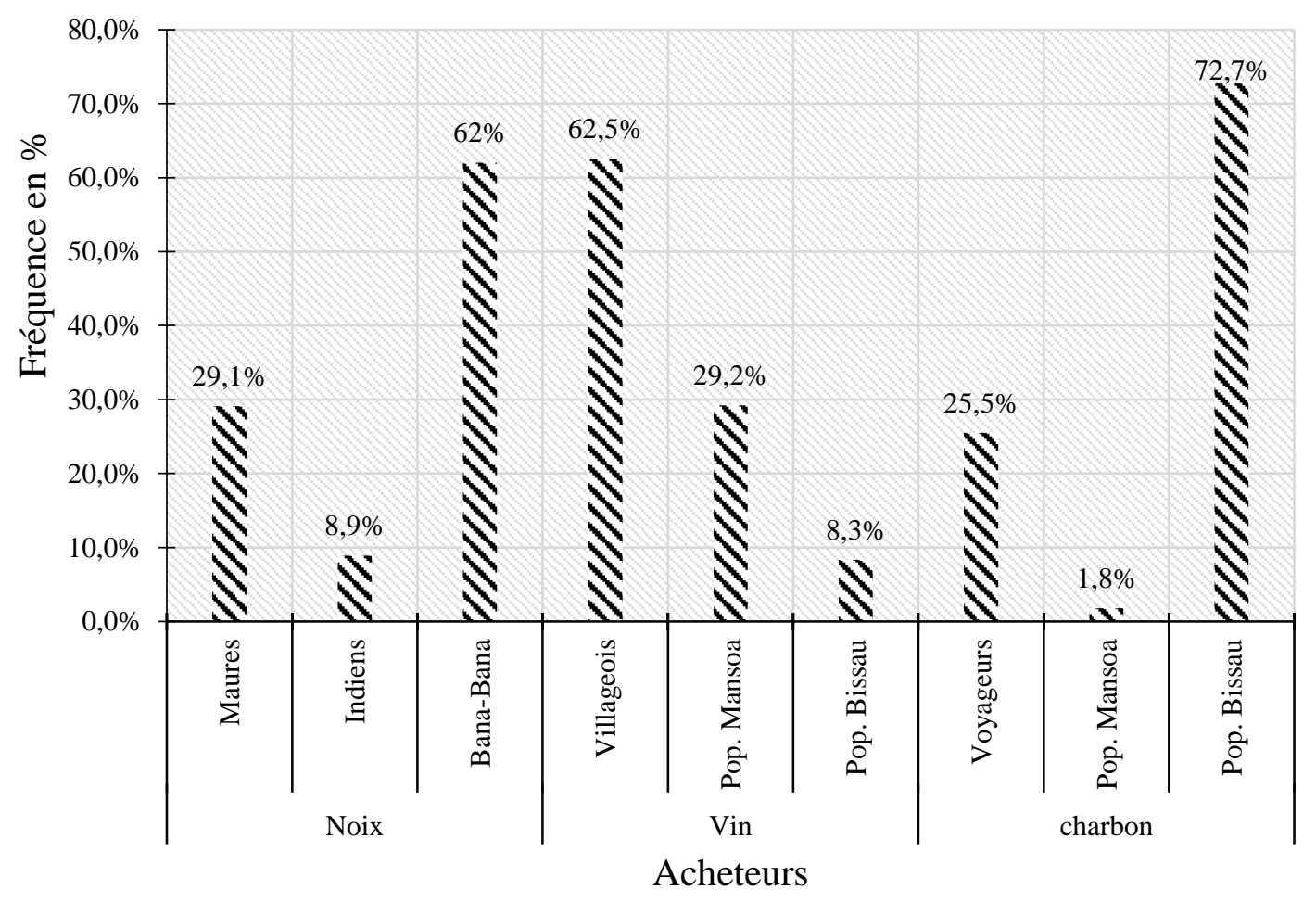

Figure 6 : Proportion des acheteurs en fonction des produits du cajou.

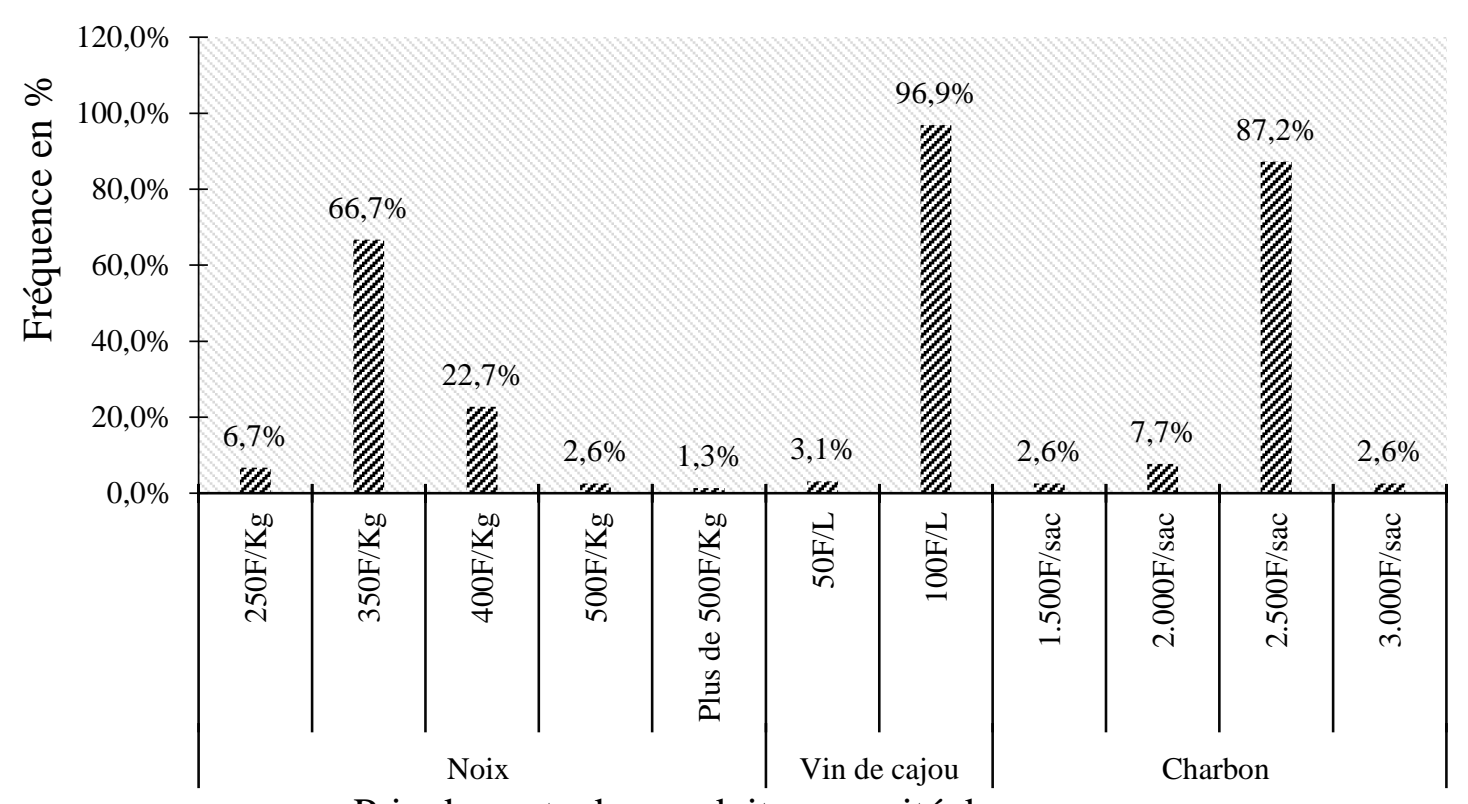

Prix de vente des produits par unité de mesure

Figure 7 : Fréquences de citation des prix de vente par unité de mesure en 2019. 
Tableau 4 : Fréquences de citation des destinations des revenus tirés du cajou.

Destinée des revenus Fréquence

Scolarité des enfants

Panneaux solaires

Construction de maison et toiture en zinc

Achat de riz

Soins médicaux de la famille

Achat de semences de riz

Achat de fertilisants des champs rizicoles

Achat de champs de riz

Paiement de la main d'œuvre des rizières

Achat de matériel de travail des rizières

Achat des animaux

Achat produits phytosanitaires (plants de riz)

$6,4 \%$

\section{DISCUSSION}

\section{Caractérisation des producteurs et tenure foncière}

L'analyse faite sur la composition de l'échantillon montre qu'elle est composée que d'hommes. Cela pourrait s'expliquer par le fait que les femmes ne participent pas à la gouvernance foncière dans ces villages où les populations appartiennent toutes à l'ethnie Balante à caractère patriarcal. Ces résultats corroborent ceux de Ribeiro (2002) qui souligne que, chez les Balantes, c'est le plus âgé des hommes qui assure, en cas de décès du père, la gestion des intérêts collectifs de la famille.

L'enquête a révélé que l'héritage (73,2\%) est le principal mode d'accès à la terre chez les Balantes. Ce résultat corrobore celui de Ribeiro (2002) qui souligne que, chez les balantes, les fils reçoivent du père de famille leurs parcelles de terre au moment où ils se marient.

\section{Mise en place des plantations d'anacarde et calendrier de production}

Dans ces zones le semis direct est le principal mode de plantation des anacardiers $(77,0 \%)$ contre $(23,0 \%)$ des plantations qui sont issues des pépinières. Le choix du semis direct pourrait s'expliquer par le fait qu'il est plus connu et plus facile à réaliser. Ce résultat reflète ceux de Balogoun et al. (2014) et ceux de Koffi et Oura (2019) qui ont montré que le semis direct est la méthode la plus utilisée respectivement au Bénin et en Côte d'Ivoire. Le défrichage/nettoyage se fait le plus souvent au mois de février $(36,4 \%)$ alors que la récolte/vente débute le mois de mars et se termine au mois de juin. Selon Ricau (2013), la production des noix d'anacarde en Guinée- 
Bissau s'étend de mars à juin avec des pics de production aux mois d'avril et de mai.

\section{Usages de la main d'œuvre dans les champs d'anacarde}

La majorité des producteurs $(94,8 \%)$ fait appel à la main d'œuvre pour le défrichage/nettoyage contre $5,2 \%$ pour la récolte/vente. La faible utilisation de la main d'œuvre pour la récolte/vente s'explique par le fait que les producteurs utilisent le plus souvent la main d'œuvre familiale. Cela pourrait aussi s'expliquer en partie par la volonté des producteurs à diminuer le coût total de la main d'œuvre. Ce résultat corrobore ceux de Balogoun et al. (2014) qui soulignent que le coût total de la main-d'œuvre a des effets négatifs et significatifs sur le revenu, ce qui fait que les femmes et les enfants sont souvent employés comme main-d'œuvre familiale.

\section{Nature et destinées des produits tirés de l'anacardier}

Les noix de cajou à l'état brutes et/ou grillées sont utilisées respectivement à $100 \%$ pour la vente et pour l'alimentation familiale dans la zone d'étude. Nos résultats confirment les travaux de Koffi et Oura (2019) qui ont montré que la vente de la noix de cajou constitue une importante source de revenus et permet d'améliorer la condition économique et le cadre de vie des ménages. Concernant le jus obtenu à partir de la pomme, il est exclusivement utilisé pour la consommation familiale dans ces villages alors que le vin est utilisé, à $47,35 \%$ des cas, pour la consommation de la famille et à $52,65 \%$ pour la vente. Selon Ndiaye et al. (2017) le jus extrait de la pomme de l'anacardier permet la fabrication du vin qui procure des revenus substantiels aux producteurs. Cependant la pomme est également utilisée $(0,6 \%)$ pour la fabrication du savon. Pour les produits ligneux, ils sont utilisés comme bois de chauffe $(52,3 \%)$ et pour faire du charbon destiné à la vente $(47,7 \%)$. Ce résultat corrobore celui de Daffé (2013) pour qui l'anacardier est une source de bois d'énergie, de charbon de bois et constitue une source de revenus non négligeable. Pour ce qui est de la pharmacopée, les feuilles, les racines et l'écorce sont utilisées avec les fréquences de citation respectives suivantes : $(7,9 \%) ;(1,3 \%)$ et $(2,6 \%)$. Certains producteurs, comme Ouzébio Nkagna, affirment que la boisson tirée d'une solution de feuilles broyées de l'anacardier permet d'arrêter la diarrhée.

\section{Commercialisation des produits de cajou}

Dans ces zones, les "bana-bana" (pisteurs) sont les principaux acheteurs de noix $(62,0 \%)$ suivi des Maures (29,1\%). Cette situation s'explique par le fait que les "banabana" sont plus proches et plus habitués aux producteurs. Pour ce qui est du vin, il est vendu dans la zone de production $(62,5 \%)$, à la population de Mansoa (29,2\%). Cela pourrait s'expliquer par le fait que le vin de cajou est très utilisé en milieu Balante. Le charbon de bois est principalement vendu à la population de Bissau $(72,7 \%)$. Ce résultat pourrait s'expliquer par le fait que le charbon est plus utilisé en ville.

\section{Destinées des revenus tirés de la production}

Au final, les revenus tirés du cajou sont destinés à l'achat du riz, du matériel de travail des champs et au paiement de la main d'œuvre rizicole avec respectivement $(14,1 \%) ;(13,4 \%)$ et $(13,2 \%)$. Ce résultat corrobore celui de Camara et al. (2007) qui souligne qu'en Guinée Bissau, le cajou permet aux paysans de se procurer du riz, principale denrée alimentaire du pays. Il en est de même pour Temudo et Abrantes (2014) pour qui, les agriculteurs investissent aussi ces revenus dans la production du riz (paiement, alimentation de la main d'œuvre etc.). Dans la même lancée, Sinan et Abou (2016) qui évoquent que l'anacarde permet aux paysans d'investir dans plusieurs domaines comme la célébration des mariages, l'achat des bœufs et/ou d'un moyen de déplacement (moto), des panneaux solaires.

\section{Conclusion}

Dans la région de notre zone d'étude, l'exploitation des champs est plus que du ressort des hommes que des femmes. L'étude qui a pour objectif d'évaluer les impacts socioéconomiques de la culture d'anacardier sur les 
producteurs des villages de Cuboi, CambiaPsora et N'Tchugal, a permis de cerner l'environnement des producteurs du secteur. La culture d'anacardier est une véritable culture de rente incontournable grâce à sa rentabilité. Les revenus engendrés par cette culture permettent aux producteurs de subvenir à leurs besoins, d'améliorer leurs conditions et cadre de vie.

En perspective, il serait intéressant de mener des études expérimentales sur les variétés d'anacarde pour pouvoir promouvoir celles qui sont plus productives en Guinée Bissau.

C'est pourquoi, dans sa politique agricole, le gouvernement bissau-guinéen devrait mettre un accent particulier pour le développement de la filière anacarde qui contribue à hauteur de 18\% PIB du pays.

\section{CONFLIT D'INTERETS}

Les auteurs déclarent, à l'unanimité, qu'il n'y a aucun conflit d'intérêts qui serait liée au présent article.

\section{CONTRIBUTIONS DES AUTEURS}

Cet article a été extrait du mémoire de fin cycle de master de MK. MK a effectué les $60 \%$ de ce travail depuis l'élaboration du protocole de recherche jusqu'à la réalisation et finalisation $\mathrm{du}$ document. $\mathrm{NN}$ en tant qu'encadreur a suivi de près ce travail depuis le début jusqu'à la finalisation du document. MD a contribué à l'amélioration de la qualité du manuscrit à travers des critiques et suggestions.

\section{REMERCIEMENTS}

Les auteurs remercient "Universsel" pour l'appui technique et financier dans le cadre du projet DEDURAM (Développement Durable de l'Agriculture de Mangrove). Nos remerciements s'adressent aussi aux producteurs d'anacarde de la zone d'étude qui ont rendu possible les enquêtes de terrain. Ils vont également à l'endroit des évaluateurs pour leur importante contribution dans l'amélioration de la qualité de mon mémoire de Master.

\section{REFERENCES}

Balogoun I, Saïdou A, Ahoton EL, Amadji LG, Ahohuendo CB, Adebo IB, Babatounde $\mathrm{S}$, Chougourou D, Adoukonou-Sagbadja H. 2014. Caractérisation des systèmes de production à base d'anacardier dans les principales zones de culture de Bénin. Agron. $\quad$ Afr., $\quad \mathbf{2 6}(1)$ : 9-22. https://www.ajol.info/index.php/aga/arti cle/view/104427

Bappah HY, Friedrich-Ebert-Stiftung, Peace and Security Centre of Competence SubSaharan Africa. 2018. Pourquoi les négociations de paix ont-elles échoué en Guinée-Bissau? Une analyse de l'économie politique de l'Accord de Conakry négocié par la CEDEAO, Guinée-Bissau, 48 p.

Benzinho J, Rosa M. 2018. À la Découverte de la Guinée-Bissau (2 ${ }^{\text {ème }}$ édn). Gráfica edilber : Coimbra, Portugal.

Cabral A. 2015. Plan Stratégique et Opérationnel 2015-2020 «Terra Ranka ». UNESCO, Guinée-Bissau, 198 p.

Camara MS, Andrade R, Dias T. 2007. Rapport final sur le commerce du cajou et du riz: implications pour la sécurité alimentaire. MAGB, Guinée Bissau, 19 p.

Daffé M. 2013. Arbres remarquables de Casamance. Ircod Alsace, Guinée Bissau, $40 \mathrm{p}$.

Ira M, Dayo GK, Sangaré M, Djassi B, Gomes J, Cassama B, Toguyéni A, Yapi-Gnaoré CV, Ouédraogo GA. 2019. Paramètres démographiques et productivité des élevages bovins de la Guinée-Bissau. Int. J. Biol. Chem. Sci., 13(2): 704-719. DOI : https://dx.doi.org/10.4314/ijbcs.v13i2.11

Koffi SY, Oura KR. 2019. Les facteurs de l'adoption de l'anacarde dans le bassin cotonnier de Côte d'Ivoire. Cah. Agric., 28: 24.

DOI: https://doi.org/10.1051/cagri/2019025

Lo MM, Barbosa MA, Cunha MBK. 2016. Etude diagnostique sur l'intégration du commerce EDIC II. EDIC II, Guinée Bissau, 308 p. 
MADR. 2010. Programme d'Action National de Lutte Contre la Désertification en Guinée-Bissau (PAN/LCD). MADR, Guinée-Bissau, 146 p.

MSI. 2010. $1^{\text {er }}$ Rapport national sur la mise en œuvre de la stratégie de Maurice +5 . MSI, Guinée-Bissau, 35 p.

Ndiaye S, Charahabil MM, Diatta M. 2017. Caractérisation des Plantations à base d'anacardier (Anacardium occidentale L.) dans le Balantacounda: cas des communes de Kaour, Goudomp et Djibanar (Casamance/Sénégal). Eur. Sci. J., $\quad$ 13: $242 . \quad$ DOI: https://doi.org/10.19044/esj.2017.v13n12 p242

NEPAD. 2006. Profil de projet d'investissement bancable «unités de transformation, conservation et emballage de noix de cajou, fruits et légumes ». NEPAD/FAO, Guinée Bissau, $18 \mathrm{p}$.

Pennober G. 1999. Analyse spatiale de l'environnement côtier de l'Archipel des Bijagos, Guinée-Bissau. Ph.D Thesis, Institut Universitaire Européen de la Mer, Université de Bretagne occidentale, 193 p.

Ribeiro CR. 2002. Conflits et gestion foncière en Guinée- Bissau. FOA, Rome, 16 p.
Ricau P. 2013. Connaître et comprendre le marché international de l'anacarde. RONGEAD, Guinée- Bissau, 49 p.

Saïdou A, Tossou R, Kossou D, Sambieni S, Richards P, Kuyper TW. 2007. Land tenure and sustainable soil fertility management in Benin. Int. J. Agri. Sust., 5(2 \& 3): 195-212.

Sinan A, Abou NK. 2016. Impacts SocioEconomiques de la Culture de l'anacarde dans la Sous-Prefecture D'odienne (Côte d'Ivoire). Eur. Sci. J., 12: 369. DOI: https://doi.org/10.19044/esj.2016.v12n32 p369

Temudo MP, Abrantes M. 2014. The Cashew Frontier in Guinea-Bissau, West Africa: Changing Landscapes and Livelihoods. Hum. Ecol., 42: 217-230. DOI: https://doi.org/10.1007/s10745-0149641-0

Universsel. 2018. Etude socio-économique de référence-livrable 4 : Note filière de riz de mangrove en Guinée-Bissau et en région Oio. DEDURAM, Guinée-Bissau, 33 p.

Yabi L, Yabi BF, Dadegnon S. 2013. Diversité des espèces végétales au sein des agroforêts à base d'anacardier dans la commune de Savalou au Benin. Int. J. Biol. Chem. Sci., 7(2): 696-706. 